Series A

\author{
I. MATHEMATICA
}

407

\title{
SUR LES ÉLÉMENTS COMPACTS D'UNE ALGÈBRE NORMÉE
}

PAR

KLAUS VALA

HELSINKI 1967

S U O M A L I NEN TIEDEAKATEM I A

doi:10.5186/aasfm.1967.407 
Présenté à l'Académie le 10 mars 1967 par MM. Oldi Lento et K. I. Virtanen 


\section{Introduction}

Soit $E$ un espace vectoriel normé. Désignons par $L(E)$ l'algèbre des applications linéaires continues de $E$ dans $E$. La topologie uniforme de $L(E)$ est engendrée par la norme usuelle qui définit sur $L(E)$ une structure d'algèbre normée. En dehors de cela, nous considérons sur $L(E)$ la structure sous-jacente d'espace vectoriel normé.

Soient maintenant $A, B \in L(E)$ et considérons l'application linéaire $T \rightarrow A^{\prime} T^{\prime} B$ de l'espace vectoriel normé $L(E)$ dans lui-même. Auparavant l'auteur a prouvé ([4], p. 6$)$ un théorème dont le cas particulier est le suivant: Soient $A \neq 0$ et $B \neq 0$; pour que l'application linéaire $T \rightarrow A T B$ soit précompacte, il faut et il suffit que les applications $A$ et $B$ soient toutes les deux précompactes. Il en découle immédiatement le corollaire suivant: Pour que l'application linéaire $T \rightarrow A T A$ de $L(E)$ dans $L(E)$ soit précompacte, il faut et il suffit que $A$ soit une application précompacte de $E$ dans $E$.

Egalement, on peut se poser la question sur les conditions sous lesquelles l'application linéaire $T \rightarrow A T B$ soit de rang fini. Si $A \neq 0$ et $B \neq 0$, une condition nécessaire et suffissante est que les applications $A$ et $B$ soient toutes les deux de rang fini, comme on peut facilement démontrer. Encore plus particulièrement, pour que l'application linéaire $T \rightarrow A T A$ soit de rang fini, il faut et il suffit que $A$ soit de rang fini.

Ces théorèmes nous ont conduits aux définitions des éléments compacts et de rang fini données au-dessous. Il faut noter que la définition d'un élément compact dans une algèbre normée commutative donnée dans [2] diffère essentiellement de la nôtre.

\section{Préliminaires}

Une algèbre normée $R$ est une algèbre associative munie d'une fonction $x \rightarrow\|x\|$ telle que $R$ (considéré comme espace vectoriel) soit un espace vectoriel normé et telle que $\|x y\| \leq\|x\|\|y\|$, pour tous $x, y \in R$. Le corps de base de $R$ peut être le corps réel ou le corps complexe; $R$ peut avoir un élément unité ou non. Nous disons qu'une application de $R$ dans $R$ est un opérateur dans $R$, si elle est linéaire et continue pour la 
structure d'espace vectoriel de $R$. Une application linéaire d'un espace vectoriel normé dans un autre est dite précompacte, si elle transforme la boule unité en un ensemble précompact. Les opérateurs précompacts dans un espace vectoriel normé $E$ forment un idéal bilatère fermé dans l'algèbre $L(E)$ de tous les opérateurs dans $E$.

\section{Les éléments compacts et les éléments de rang fini de $\mathbf{R}$.}

Soit $R$ une algèbre normée et $a$ un élément de $R$. Désignons par $\beta(a)$ l'application $x \rightarrow$ axa de $R$ dans $R$. L'application $\beta(a)$ est évidemment linéaire et continue. Si cet opérateur est précompact, nous disons que $a$ est un élément compact de $R$. Désignons par $C$ l'ensemble de tous les éléments compacts de $R$. Nous disons qu'un élément $a \in R$ est de rang fini, si l'opérateur $\beta(a)$ est de rang fini et nous désignons par $F$ l'ensemble de tous ces éléments. Evidemment nous avons $F \subset C$.

Il peut arriver que $F=C=R$; l'algèbre $L(E)$, où $E$ est un espace vectoriel normé de dimension finie, en donne un exemple. Mais il peut aussi arriver que $F=C=\{0\}, R \neq\{0\}$. Il est ainsi, par exemple, dans le cas où $R$ est l'algèbre des fonctions réels continues dans l'intervalle $[0,1]$ et normées par la métrique de la convergence uniforme (cf. [2] p. 280). Pour avoir un exemple où toutes les inclusions dans $\{0\} \subset F \subset C \subset R$ sont propres, on peut prendre pour $R$ l'algèbre $L(E)$, où $E$ est un espace hilbertien de dimension infinie.

Proposition 1. L'ensemble $C$ est fermé.

Soit $a \in \bar{C}$ et $\left(a_{k}\right)$ une suite telle que $a_{k} \in C$ et $a_{k} \rightarrow a$, pour $k \rightarrow \infty$. Nous avons pour un $x \in R$ arbitraire:

$$
\begin{aligned}
& \left\|a_{k} x a_{k}-a x a\right\|=\left\|\left(a_{k}-a\right) x a_{k}+a x\left(a_{k}-a\right)\right\| \\
& \leq\left\|\left(a_{k}-a\right) x a_{k}\right\|+\left\|a x\left(a_{k}-a\right)\right\| \\
& \leq\left(\left\|a_{k}-a\right\|\left\|a_{k}\right\|+\|a\|\left\|a_{k}-a\right\|\right)\|x\| \leq M\left\|a_{k}-a\right\|\|x\|,
\end{aligned}
$$

où $M$ est un constant. Il en découle que $\left\|\beta\left(a_{k}\right)-\beta(a)\right\| \rightarrow 0$, pour $k \rightarrow \infty$. Comme les $\beta\left(a_{k}\right)$ sont précompacts, il en résulte que $\beta(a)$ est précompact et donc, par la définition, $a$ est un élément compact.

Corollaire. L'adhérence $\bar{F}$ de $F^{\prime}$ est contenue dans $C$.

Proposition 2. Tout élément compact idempotent de $R$ est de rang fini.

En effet, de $a^{2}=a$ il découle que $a^{2} x a^{2}=a x a$, pour tous $x \in R$, ou $\beta(a)^{2}=\beta(a)$; comme l'opérateur $\beta(a)$ est précompact cela entraîne qu'il est de rang fini. 
Proposition 3. Si $a$ est un élément compact (resp. de rang fini) et $u$ un élément arbitraire de $R$, les éléments au et $u a$ sont compacts (resp. de rang fini).

Les applications $x \rightarrow$ auxau et $x \rightarrow$ uaxua sont toutes les deux composées de trois opérateurs dont un est $\beta(a)$. La proposition résulte alors du fait que les opérateurs précompacts (resp. de rang fini) de $R$ forment un idéal bilat ère.

\section{Un exemple}

Si $a$ et $b$ sont tous les deux des éléments compacts (resp. de rang fini), l'opérateur $x \rightarrow a x b$ n'est pas nécessairement précompact (resp. de rang fini); nous allons en donner un exemple.

Soit donc $E=E_{1} \oplus E_{2}$, où $E_{1}$ et $E_{2}$ sont des espaces hilbertiens proprement dits, c'est à dire, isomorphes à $l^{2}$, et soit $R=\{T \in L(E) \mid$ $\left.T(E) \subset E_{2}\right\}$. Choisissons une projection $A \in R$ de rang fini et $\neq\{0\}$ et $B \in R$ tel que $B\left(E_{1}\right)=E_{2}, B$ est une isométrie dans $E_{1}$ et $B\left(E_{2}\right)=\{0\}$. L'application $T \rightarrow A T A$ est de rang fini et l'application $T \rightarrow B T B$ est identiquement $=0$, donc aussi de rang fini. Dans le sens de la définition dans le n:o précédant, $A$ et $B$ sont donc tous les deux des éléments de rang fini de $R$ et à fortiori des éléments compacts de $R$. Mais l'application $T \rightarrow A T B$ de $R$ dans $R$ n'est pas précompacte, comme on peut aisement vérifier directement ou sur la base du théorème 3 en [4].

\section{Sur certains idéaux dans $\mathbf{R}$}

La proposition 3 exprime le fait que les ensembles $C$ et $F$ sont stables pour la multiplication dans $R$. Mais, en général, ils ne sont pas des idéaux, comme le montre l'exemple dans le n:o précédant.

Convenons de désigner par $C_{0}$ (resp. par $F_{0}$ ) l'ensemble de tous les éléments compacts (resp. de rang fini) $a \in R$, tels que les applications linéaires $x \rightarrow a x b$ et $x \rightarrow b x a$ de $R$ dans $R$ sont précompactes (resp. de rarg fini) pour tous $b \in C^{\prime}$ (resp. pour tous $b \in F$ ). En s'appuyant sur le fait que les opérateurs précompacts dans $R$ forment un ensemble fermé on démontre facilement que $C_{0}$ est fermé.

Proposition 4. $C_{0}$ et $F_{0}$ sont des idéaux dans $R$.

Soient $a, a^{\prime} \in C_{0} \quad$ (resp. $a, a^{\prime} \in F_{0}$ ), $b \in C$ et $u \in R$; puisque les opérateurs précompacts (resp. de rang fini) forment un idéal bilatère, on vérifie immédiatement que toutes les applications linéaires $x \rightarrow a u x b$, 
$x \rightarrow b x a u, x \rightarrow$ uaxb, $x \rightarrow b x u a, x \rightarrow\left(a+a^{\prime}\right) x b$ et $x \rightarrow b x\left(a+a^{\prime}\right)$ sont précompactes (resp. de rang fini), d'où la proposition.

Soit $H$ un idéal dans $R$; nous convenons de dire que $H$ est un $c$-idéal (resp. un $f$-idéal), si l'opérateur $x \rightarrow a x b$ dans $R$ est un opérateur précompact (resp. de rang fini), pour tous $a, b \in H$. Evidemment, tout $c$-idéal est contenu dans $C$ et tout $f$-idéal est contenu dans $F$. Les idéaux $C_{0}$ et $F_{0}$ considérés au-dessus sont des exemples de c-idéaux, resp. de $f$-idéaux. La proposition qui suit en donne d'autres exemples.

Proposition 5. Si $a$ est un élément compact de $R$ (resp. un élément de rang fini de $R$ ), l'idéal bilatère $H(a)$ engendré par $a$ est un $c$-idéal (resp. un $f$-idéal).

Pour la démonstration il faut observer que tout élément de $H(a)$ peut se mettre sous la forme

$$
n a+u a+a v+\sum_{k} u_{k} a v_{k}
$$

où $n$ est un nombre entier et $u, v, u_{k}, v_{k} \in R$. Si $a \in C$ (resp. $a \in F$ ) et $z_{1}, z_{2} \in H(a)$, l'application linéaire $x \rightarrow z_{1} x z_{2}$ peut donc se mettre sous la forme d'une somme, où chaque membre est une application composée dont un facteur est l'opérateur $\beta(a)$. Puisque les opérateurs précompacts (resp. de rang fini) dans $R$ forment un idéal bilatère, il en résulte que l'opérateur $x \rightarrow z_{1} x z_{2}$ est précompact (resp. de rang fini). La proposition est ainsi démontrée.

Proposition 6. Quel que soit le $c$-idéal (resp. $f$-idéal) $H$ dans $R$, il existe un $c$-idéal (resp. un $f$-idéal) maximal $J$ contenant $H$.

En effet, on peut facilement vérifier que l'ensemble ordonné des $c$ idéaux (resp. des $f$-idéaux) contenant $H$ est inductif; la proposition est ainsi une conséquence du théorème de Zorn.

Proposition 7. Pour qu'il existe un seul $c$-idéal (resp. $f$-idéal) maximal dans $R$, il faut et il suffit que $C$ soit un $c$-idéal (resp. que $F$ soit un $f$-idéal).

La suffisance de la condition étant évidente, nous supposons que $J$ est l'unique $c$-idéal (resp. $f$-idéal) maximal. Pour tous $a \in C$ (resp. $a \in F^{\prime}$ ) l'idéal $H(a)$ est un $c$-idéal (resp. un $f$-idéal), d'après la proposition 5 ; donc $H(a) \subset J$. En conséquence

$$
\left.C=\bigcup_{a \in C} H(a) \subset J \text { (resp. } F=\bigcup_{a \in F} H(a) \subset J\right)
$$

Comme $J \subset C$ (resp. $J \subset F$ ), cela entraine que $C=J$ (resp. $F=J$ ), donc $C$ est un $c$-idéal (resp. un $f$-idéal).

On peut noter que dans le cas où $C$ (resp. $F$ ) est un $c$-idéal (resp. un $f$-idéal), il est identique à l'idéal $C_{0}$ (resp. $F_{0}$ ), considéré plus haut. 


\section{Sur certains annulateurs dans $\mathbf{R}$}

On sait que pour un opérateur précompact $A$ le noyau de $I-A$ ( $I$ est l'opérateur identique) est de dimension finie et le rang de $I-A$ est de codimension finie. Pour une algèbre normée arbitraire $R$, nous avons les résultats suivants.

Proposition 8. Soit $a$ un élément compact dans $R$, et soit $e$ un élément idempotent de $R$, tel que $e=a e$ ou $e=e a$; alors $e$ est de rang fini.

En effet, ea et ae sont des éléments compacts d'après la proposition 3 ; cela entraîne, en vertu de la proposition 2 , que $e$ est de rang fini.

Proposition 9. Soit $a$ un élément compact de $R$ et soit $u$ un élément de $R$ tel que $u=a u=u a$; alors $u$ est de rang fini; la dimension du sous-espace vectoriel $\{u \in R \mid u=u a=a u\}$ est finie.

Pour tous $x \in R$ nous avons, d'après l'hypothèse, $a(u x u) a=u x u$, donc le rang de l'opérateur $\beta(u)$ est invariant sous l'opérateur $\beta(a)$; en conséquence, c'est un sous-espace vectoriel de dimension finie, puisque $\beta(a)$ est précompact. Cela signifie que $u$ est de rang fini.

Pour tous $u \in R$ tels que $u=a u=u a$, nous avons $a u a=u$. L'espace vectoriel formé par ces éléments est donc invariant sous $\beta(a)$; en conséquence sa dimension est finie.

Proposition 10. Soit $R$ une algèbre normée avec élément unité noté par l et soit $a$ un élément compact de $R$. Notons par $N_{k}$ le sous-espace vectoriel de $R$ formé par les éléments $u$ tels que $a u=u a$ et $(1-a)^{k} u=0$ $(k=1,2, \ldots)$. Il existe alors un nombre naturel $p$ tel que $N_{k}=N_{p}$, pour tous $k>p$.

Supposons qu'un tel $p$ n'existe pas; cela signifie que $N_{1} \subset N_{2} \subset \cdots$ $\subset N_{k} \subset \cdots$, où toutes les inclusions sont propres. Par un lemme de Riesz ([3], p. 96), nous pouvons choisir dans chaque $N_{\dot{k}}$ un élément $x_{k}$, tel que $\left\|x_{k}\right\|=1$ et $\left\|x_{k}-u\right\| \geq \frac{1}{2}$, pour tous $u \in N_{k-1}$. Soit $i<k$; posons $v=x_{k}-a x_{k} a+a x_{i} a=\left(1-a^{2}\right) x_{k}+a x_{i} a=(1-a)(1+a) x_{k}+a x_{i} a$. Alors, $v \in N_{k-1}$, et, en conséquence,

$$
\left\|x_{k}-v\right\|=\left\|a x_{k} a-a x_{i} a\right\| \geq \frac{1}{2},
$$

qui est absurde, puisque l'opérateur $\beta(a)$ est compact. La proposition se trouve ainsi démontrée. 


\section{Bibliographie}

[1] Banach, S.: Théorie des opérations linéaires. - Warszawa, 1932.

[2] Freundlich, M.: Completely continuous elements of a normed ring. - Duke Math. J. $16,1949$.

[3] Taylor, A. E.: Introduction to functional analysis. - Wiley, New York, 1958.

[4] Vala, K.: On compact sets of compact operators. - Ann. Acad. Sci. Fenn. A.I. 351, 1964. 